\title{
A LIMIT INVOLVING FUNCTIONS IN $W_{0}^{1, p}(\Omega)$ \\ BY \\ BIAGIO RICCERI (CATANIA)
}

Abstract. We point out the following fact: if $\Omega \subset \mathbb{R}^{n}$ is a bounded open set, $\delta>0$, and $p>1$, then

$$
\lim _{\varepsilon \rightarrow 0^{+}} \inf _{u \in V_{\varepsilon}} \int_{\Omega}|\nabla u(x)|^{p} d x=\infty,
$$

where $V_{\varepsilon}=\left\{u \in W_{0}^{1, p}(\Omega): \operatorname{meas}(\{x \in \Omega:|u(x)|<\delta\})<\varepsilon\right\}$.

Here and in the sequel, $\Omega \subset \mathbb{R}^{n}$ is a (non-empty) bounded open set, $m$ denotes the Lebesgue measure in $\mathbb{R}^{n}, \delta>0, p>1$, and $W_{0}^{1, p}(\Omega)$ is the usual Sobolev space, equipped with the norm $\|u\|=\left(\int_{\Omega}|\nabla u(x)|^{p} d x\right)^{1 / p}$.

The aim of this paper is to prove the following result which could be useful in certain cases:

TheOrem 1. For each $\varepsilon>0$, put

$$
V_{\varepsilon}=\left\{u \in W_{0}^{1, p}(\Omega): m(\{x \in \Omega:|u(x)|<\delta\})<\varepsilon\right\} .
$$

Then

$$
\lim _{\varepsilon \rightarrow 0^{+}} \inf _{u \in V_{\varepsilon}} \int_{\Omega}|\nabla u(x)|^{p} d x=\infty .
$$

Before giving the proof of Theorem 1, we establish the following proposition:

Proposition 1. For each $u \in W_{0}^{1, p}(\Omega)$,

$$
m(\{x \in \Omega:|u(x)|<\delta\})>0 .
$$

Pr o of. For simplicity, let us introduce some notation. We first put

$$
\Gamma=\{x \in \Omega:|u(x)|<\delta\} .
$$

We think of $\Omega$ as a subset of $\mathbb{R} \times \mathbb{R}^{n-1}$. If $x \in \mathbb{R}^{n}$, we set $x=(t, \xi)$, where $t \in \mathbb{R}$ and $\xi \in \mathbb{R}^{n-1}$. We also denote by $A$ (resp. $B$ ) the projection of $\Omega$ on $\mathbb{R}$ (resp. $\mathbb{R}^{n-1}$ ), and by $m_{1}$ (resp. $m_{n-1}$ ) the Lebesgue measure on $\mathbb{R}$ (resp.

1991 Mathematics Subject Classification: Primary 46E35. 
$\mathbb{R}^{n-1}$ ). So, $A$ and $B$ are (non-empty) open sets, and hence $m_{1}(A)>0$ and $m_{n-1}(B)>0$. Finally, for a generic set $S \subseteq \Omega$ and for each $\xi \in B$, put

$$
S_{\xi}=\{t \in A:(t, \xi) \in S\} .
$$

By well-known results ([1], [2]), we can assume that, for almost every $\xi \in B$, the function $u(\cdot, \xi)$ belongs to $W_{0}^{1, p}\left(\Omega_{\xi}\right)$, and so it is almost everywhere equal to a function which is continuous in $\bar{\Omega}_{\xi}$ and zero on $\partial \Omega_{\xi}$. Consequently, we have $m_{1}\left(\Gamma_{\xi}\right)>0$ a.e. in $B$. Now, if $\chi_{\Gamma}$ denotes the characteristic function of $\Gamma$, then Fubini's theorem yields

$$
m(\Gamma)=\int_{A \times B} \chi_{\Gamma}(t, \xi) d t d \xi=\int_{B}\left(\int_{\Gamma_{\xi}} d t\right) d \xi=\int_{B} m_{1}\left(\Gamma_{\xi}\right) d \xi>0,
$$

as claimed.

Proof of Theorem 1. Clearly, the function $\varepsilon \mapsto \inf _{u \in V_{\varepsilon}} \int_{\Omega}|\nabla u(x)|^{p} d x$ is non-increasing. Consequently,

$$
\lim _{\varepsilon \rightarrow 0^{+}} \inf _{u \in V_{\varepsilon}} \int_{\Omega}|\nabla u(x)|^{p} d x=\sup _{\varepsilon>0} \inf _{u \in V_{\varepsilon}} \int_{\Omega}|\nabla u(x)|^{p} d x .
$$

Arguing by contradiction, assume that there is $M>0$ such that

$$
\inf _{u \in V_{\varepsilon}} \int_{\Omega}|\nabla u(x)|^{p} d x<M
$$

for all $\varepsilon>0$. Consider the function $g: \mathbb{R} \rightarrow \mathbb{R}$ defined by

$$
g(t)= \begin{cases}\delta-|t| & \text { if }|t|<\delta \\ 0 & \text { if }|t| \geq \delta\end{cases}
$$

Consider also the functional $\Psi: W_{0}^{1, p}(\Omega) \rightarrow \mathbb{R}$ defined by putting

$$
\Psi(u)=\int_{\Omega} g(u(x)) d x
$$

for all $u \in W_{0}^{1, p}(\Omega)$. Using the Rellich-Kondrashov theorem, one sees that $\Psi$ is sequentially weakly continuous in $W_{0}^{1, p}(\Omega)$. Now, for each $h \in \mathbb{N}$, choose $u_{h} \in V_{1 /(h \delta)}$ in such a way that

$$
\int_{\Omega}\left|\nabla u_{h}(x)\right|^{p} d x<M .
$$

So, the sequence $\left\{u_{h}\right\}$ is bounded in $W_{0}^{1, p}(\Omega)$. Consequently, since $p>1$, there is a subsequence $\left\{u_{h_{k}}\right\}$ weakly converging to some $u_{0} \in W_{0}^{1, p}(\Omega)$. For each $k \in \mathbb{N}$, we have

$$
\Psi\left(u_{h_{k}}\right)=\int_{\left\{x \in \Omega:\left|u_{h_{k}}(x)\right|<\delta\right\}}\left(\delta-\left|u_{h_{k}}(x)\right|\right) d x<\frac{1}{h_{k} \delta} \delta=\frac{1}{h_{k}} .
$$


Passing to the limit as $k \rightarrow \infty$, we then get $\Psi\left(u_{0}\right)=0$. This implies that $m\left(\left\{x \in \Omega:\left|u_{0}(x)\right|<\delta\right\}\right)=0$, contrary to Proposition 1 .

For $p=1$, we have the following result:

Theorem 2. Let $n=1$. For each $\varepsilon>0$, put

$$
U_{\varepsilon}=\left\{u \in W_{0}^{1,1}(\Omega): m(\{x \in \Omega:|u(x)|<\delta\})<\varepsilon\right\} .
$$

If $k$ denotes the number (possibly infinite) of connected components of $\Omega$, then

$$
\lim _{\varepsilon \rightarrow 0^{+}} \inf _{u \in U_{\varepsilon}} \int_{\Omega}\left|u^{\prime}(x)\right| d x=2 k \delta .
$$

Proof. First, assume that $k$ is finite. Let $] a_{i}, b_{i}[(i=1, \ldots, k)$ denote the connected components of $\Omega$. Suppose that $\varepsilon \leq \min _{1<i<k}\left(b_{i}-a_{i}\right)$. Let $v \in U_{\varepsilon}$. We can assume that $v$ is absolutely continuous in each interval $\left[a_{i}, b_{i}\right]$. Fix $i$. Since $v\left(a_{i}\right)=v\left(b_{i}\right)=0$, due to the choice of $\varepsilon$, there is $\left.x_{i} \in\right] a_{i}, b_{i}$ [ such that $\left|v\left(x_{i}\right)\right|=\delta$. Assume, for instance, that $v\left(x_{i}\right)=\delta$. Then

$$
\delta=\int_{a_{i}}^{x_{i}} v^{\prime}(x) d x \leq \int_{a_{i}}^{x_{i}}\left|v^{\prime}(x)\right| d x
$$

and

$$
\delta=-\int_{x_{i}}^{b_{i}} v^{\prime}(x) d x \leq \int_{x_{i}}^{b_{i}}\left|v^{\prime}(x)\right| d x
$$

Hence,

$$
2 \delta \leq \int_{a_{i}}^{b_{i}}\left|v^{\prime}(x)\right| d x .
$$

With obvious changes, one gets this inequality also if $v\left(x_{i}\right)=-\delta$. Consequently,

$$
2 k \delta \leq \sum_{i=1}^{k} \int_{a_{i}}^{b_{i}}\left|v^{\prime}(x)\right| d x=\int_{\Omega}\left|v^{\prime}(x)\right| d x .
$$

We then infer that

$$
2 k \delta \leq \inf _{u \in U_{\varepsilon}} \int_{\Omega}\left|u^{\prime}(x)\right| d x .
$$

Now, consider the function $w: \Omega \rightarrow \mathbb{R}$ defined by

$$
w(x)= \begin{cases}4 k \delta\left(x-a_{i}\right) / \varepsilon & \text { if } \left.x \in] a_{i}, a_{i}+\varepsilon /(4 k)\right], \\ \delta & \text { if } x \in] a_{i}+\varepsilon /(4 k), b_{i}-\varepsilon /(4 k)[, \\ 4 k \delta\left(b_{i}-x\right) / \varepsilon & \text { if } x \in\left[b_{i}-\varepsilon /(4 k), b_{i}[.\right.\end{cases}
$$


Clearly, $w \in U_{\varepsilon}$. Moreover, a simple calculation gives $\int_{\Omega}\left|w^{\prime}(x)\right| d x=2 k \delta$. This and (1) then show that

$$
\inf _{u \in U_{\varepsilon}} \int_{\Omega}\left|u^{\prime}(x)\right| d x=2 k \delta .
$$

Therefore, our conclusion is proved when $k$ is finite.

Now, assume that $\Omega$ has infinitely many connected components. Let $r \in \mathbb{N}$. Let $] \alpha_{i}, \beta_{i}[(i=1, \ldots, r)$ be $r$ distinct connected components of $\Omega$. Fix $\varepsilon \leq \min _{1 \leq i \leq r}\left(\beta_{i}-\alpha_{i}\right)$, and let $v \in U_{\varepsilon}$. Then, from the first part of the proof, we know that

$$
2 r \delta \leq \sum_{i=1}^{r} \int_{\alpha_{i}}^{\beta_{i}}\left|v^{\prime}(x)\right| d x \leq \int_{\Omega}\left|v^{\prime}(x)\right| d x .
$$

Hence,

$$
2 r \delta \leq \inf _{u \in U_{\varepsilon}} \int_{\Omega}\left|u^{\prime}(x)\right| d x .
$$

This, of course, implies that

$$
\lim _{\varepsilon \rightarrow 0^{+}} \inf _{u \in U_{\varepsilon}} \int_{\Omega}\left|u^{\prime}(x)\right| d x=\infty,
$$

and the proof is complete.

\section{REFERENCES}

[1] H. Brézis, Analyse fonctionnelle, Masson, 1983.

[2] V. G. Maz'ja, Sobolev Spaces, Springer, 1985.

Department of Mathematics

University of Catania

Viale A. Doria 6

95125 Catania, Italy

E-mail: ricceri@dipmat.unict.it

Received 21 June 1999 